\title{
Light interaction with multilayer arbitrary anisotropic structure: an explicit analytical solution and application for sub- wavelength imaging
}

\author{
Yasaman Kiasat, ${ }^{1,2,{ }^{*}}$ Zsolt Szabo, ${ }^{3}$ Xudong Chen, ${ }^{2}$ Erping $\mathbf{L i}^{1}$ \\ ${ }^{1}$ Institute of High Performance Computing, Agency for Science and Technology, 1 Fusionopolis Way, \#16-16 Connexis North, Singapore \\ 138632 \\ ${ }^{2}$ National University of Singapore, Department of Electrical and Computer Engineering \\ 4 Engineering Drive 3 Singapore 117583 \\ ${ }^{3}$ Budapest University of Technology and Economics, Department of Electrical Engineering 1111 Budapest, Technical University rkp, \\ Hungary \\ *Corresponding author: g0900296@nus.edu.sg
}

Received Month X, XXXX; revised Month X, XXXX; accepted Month X, XXXX; posted Month X, XXXX (Doc. ID XXXXX); published Month X, XXXX

\begin{abstract}
A systematic analytical approach to simulate the propagation of electromagnetic plane waves in multilayer anisotropic structures, where the layers can have arbitrary oriented optical axis is presented. The explicit expressions for the vector polarizations of electric and magnetic fields inside a randomly oriented anisotropic medium are derived. The developed algorithm operates with analytic $4 \times 4$ matrices to calculate the transmission and reflection coefficients. This algorithm is suitable to investigate the near-field/far-field electromagnetic wave interaction at any angle of incidence for numerous intriguing applications. The procedure is applied to design anisotropic single and multilayer lenses for sub-wavelength imaging.
\end{abstract}

OCIS codes: (260.0260) Physical optics;(260.1140) Bifringence; (310.4165) Multilayer design; (310.5446) Polarization and other optical properties (310.6628) Subwavelength structure, nanostructure; (350.5500) Propagation

\section{Introduction}

The state of the art technology of the nano-fabrication facilitates the science and engineering society to implement intriguing applications with multilayer anisotropic structures. Novel layered anisotropic structures are applied in material science [1], electroanalytical chemistry $[2,3]$, biological interfaces and tissue engineering $[4,5]$, physics and optics [1]. To characterize the microscopic structural changes in these thin films [1-5], various techniques such as x-ray reflectivity, Raman spectroscopy, fluorescent spectroscopy, optical ellipsometric spectroscopy, and infrared reflection spectroscopy are used [6]. The functionality of these techniques depends on the propagation of the light in thin films. Usually the electric field component of the light interacts with the sample. This interaction is governed by the dielectric functions of the material and the sample geometry. The properties and the performance of the sample is then obtained by the information gained from the reflected and/or transmitted field. Consequently, a clear and relatively simple analytical approach that can derive the required information from the reflected or transmitted spectra is required.

Although the analytical investigation for electromagnetic wave propagation in anisotropic layered media has been a subject of interest for many years [7-18], the presented solutions are either not systematic enough for the treatment of general multilayer birefringent media [7-11], or in the case of general solutions, the solution becomes singular for isotropic layers [12, 14]. The general solution offered in [13] is involved with power series expansions and no explicit expressions are provided for the interaction of the wave with the incident and exit medium. In [16], the wave propagation is treated in more detail, but only for a single uniaxial layer. None of the papers [7-18] provides explicit expressions for the polarization of the electric and magnetic fields in each layer. The explicit expressions for the polarization of the electric field, magnetic field, and the wave vector [14] in each layer, provides accurate information about the behavior of the electromagnetic wave propagating through layered structure for different applications [7-11, 13-15] and how the layered structure eventually transmits and reflects the incident wave [1214]. Moreover, the polarization-dependent optical investigations have become standard methods to explore the properties of anisotropic solids and liquids [17-19], and hence it is important to derive analytical expressions for the polarizations in each layer for characterization purposes. 
The paper is organized as follow. In the first part, based on the full-wave solution of the Maxwell equations, we present the explicit expressions of the electromagnetic field components in a multilayer with arbitrary oriented optical axis. In section II, the Maxwell equations are solved in the $\mathrm{k}$-space to find the explicit expressions of the partial fields. As it is convenient to reduce the number of electromagnetic field variables to a minimum, the six components of the $\mathrm{E}^{-}$ field and $\mathrm{H}$-field in each medium are expressed in terms of only one component. In section III a brief review of monochromatic plane wave propagation in layered structures is presented. Based on the analytical expressions of vector field's polarizations in section II, the boundary condition and propagation matrices are introduced for each layer, as the building blocks of the transfer matrix method in this paper. The methodology is suitable to calculate the transfer matrix of a layer with arbitrary thickness and anisotropy, for any angle of incidence under plane wave illumination. The reflection and transmission coefficients for the multilayer system, is derived from the relations between the amplitudes of the incident, reflected, and transmitted waves. It is shown that similar boundary condition matrix relates the transfer function of the layered structure to the amplitudes of the waves in the incident and exit media. The method is suitable for propagating wave and evanescent wave calculations as well. In section IV the derived transfer function method is applied to calculate the transmission of images with propagating and evanescent components with sub-wavelength details, through anisotropic single or multilayer flat lenses. Section $V$ is allocated to the investigation of the effect of gyrotropy on sub-wavelength imaging process. In appendix $A$, it is shown that the derived anisotropic relation can be reduced to the isotropic case without any singularity in contrast to the method presented in $[12,14]$.

\section{Explicit Expressions of the Electric and Magnetic fields in Arbitrary Anisotropic Media}

In this section the polarizations of vector fields from Maxwell equations is provided. Without loss of generality a coordinate system with the $\mathrm{z}$ axis perpendicular to the multilayer structure is introduced as it is presented in Fig. 1. For a non-magnetic medium with arbitrary anisotropy, the polarizations of the fields depend on the permittivity tensor $\overline{\bar{\varepsilon}}$ and the wave vector $\mathbf{k}$ in each medium. The tangential component of the wave vector, here $k_{x}$, is conserved through the interfaces, therefore it is known in all layers. The normal component, $k_{z}$ can be obtained combining the following Maxwell equations

$$
\begin{gathered}
\nabla \times H=j \omega D \\
\nabla \times E=-j \omega \mu H
\end{gathered}
$$

Where $\mathrm{D}=\overline{\bar{\varepsilon}} E$, which results in the wave equation in $\mathrm{k}$ space. The nontrivial solution of the wave equation in the anisotropic medium is a quadratic dispersion relation that yields four roots, $(i=1-4)$. These four roots are the $\mathrm{z}$ components of the wave vectors in the anisotropic layer. The four explicit expressions for $k_{z}$ are given in [14]. Two solutions have a real positive part and constitute the forward-traveling plane waves with respect to $+z$, while the other two solutions with negative real-parts are the backpropagating waves. In order to find the explicit expressions for the E-field and $\mathrm{H}$-field polarization vectors, equations (1a) and (1b) are used. From equation (1a),

$$
\left\{\begin{array}{c}
-\frac{\partial H_{y}}{\partial z}=j \omega D_{x} \\
\frac{\partial H_{x}}{\partial z}-\frac{\partial H_{z}}{\partial x}=j \omega D_{y} \\
\frac{\partial H_{y}}{\partial x}=j \omega D_{z}
\end{array}\right.
$$

From the first and the third relations in equation 2,

$$
\begin{aligned}
& k_{z}(i) H_{y}=\omega\left(\varepsilon_{x x} E_{x}+\varepsilon_{x y} E_{y}+\varepsilon_{x z} E_{z}\right) \\
& k_{x} H_{y}=-\omega\left(\varepsilon_{z x} E_{x}+\varepsilon_{z y} E_{y}+\varepsilon_{z z} E_{z}\right)
\end{aligned}
$$

Here $\varepsilon_{n m}(n, m \in\{x, y, z\})$ is the component of the permittivity tensor connecting $D_{n}$ to $E_{m}$ where $\mathrm{D}$ and $\mathrm{E}$ are the electric displacement and electric field, respectively. The combination of Equations (3a), (3b), and (1b) results in the explicit expressions for the E-field polarization vector,

$$
\vec{E}=\left[\begin{array}{c}
1 \\
\frac{-\alpha}{\gamma}-\frac{\beta \lambda}{\xi \gamma} \\
\frac{\lambda}{\xi}
\end{array}\right] E_{x}
$$

Where $\alpha, \beta, \gamma, \xi$, and $\lambda$ are defined as

$$
\begin{gathered}
\beta_{l}=\frac{\varepsilon_{x z}}{k_{z}(i)}+\frac{\varepsilon_{z z}}{k_{x}} \\
\alpha_{l}=\frac{\varepsilon_{x x}}{k_{z}(i)}+\frac{\varepsilon_{z x}}{k_{x}} \\
\gamma_{l}=\frac{\varepsilon_{x y}}{k_{z}(i)}+\frac{\varepsilon_{z y}}{k_{x}}
\end{gathered}
$$




$$
\begin{aligned}
& \lambda_{l}=k_{x} \varepsilon_{x x}+k_{z}(i) \varepsilon_{x z}-\left(\varepsilon_{x y}-\varepsilon_{y z}\right) \frac{\alpha_{l}}{\gamma_{l}} \\
& \xi_{l}=k_{x} \varepsilon_{z x}+k_{z}(i) \varepsilon_{z z}+\left(\varepsilon_{x y}-\varepsilon_{z y}\right) \frac{\beta_{l}}{\gamma_{l}}
\end{aligned}
$$

Where ' $i$ ' refers to the numbers of the partial waves propagating in one layer. The polarization vector for $\mathrm{H}^{-}$ field is determined by

$$
\vec{H}=\frac{\nabla \times E}{-j \omega \mu}=\left[\begin{array}{c}
\frac{-k_{z}(i)}{\omega \mu}\left(\frac{-\alpha}{\gamma}-\frac{\beta \lambda}{\xi \gamma}\right) \\
\frac{1}{\mu \omega}\left(k_{z}(i)-k_{x} \frac{\lambda}{\xi}\right) \\
\frac{k_{x}}{\omega \mu}\left(\frac{-\alpha}{\gamma}-\frac{\beta \lambda}{\xi \gamma}\right)
\end{array}\right] E_{x}
$$

As is expected for arbitrary anisotropic material, the field components are coupled to each other and no TE/ TM polarization with respect to the direction of propagation is possible. In fact a plane wave in the arbitrary anisotropic medium can be decomposed into two orthogonal waves, called a-wave and $b$-wave which are generalizations of the TE and TM waves in isotropic and uniaxially anisotropic media. The vectors, $\mathbf{a}$ and $\mathbf{b}$, each with six components, are defined by the optical properties of the medium [20].

\section{General Transfer Matrix}

Consider the source plane wave propagating from the incident $(-\infty<\mathrm{z}<0)$ medium with a complex relative permittivity of $\varepsilon_{i}$, at an angle of incidence $\theta_{i}$ and $A_{i, T E}, A_{i, T M}, B_{r, T E}$, and $B_{r, T M}$ are the complex amplitudes of the TE and TM modes of the incident and reflected waves, respectively. After interaction with the layered structure, the wave enters the exit medium, $z_{n}<\mathrm{z}<\infty$ and is characterized with the complex transmission amplitude of $A_{t, T E}$ and $A_{t, T M}$ for transmitted TE and TM polarization respectively. The propagation occurs along the $\mathrm{z}$ direction and the layers are in the $x^{-} z$ plane. The origin is set at the plane which constitutes the interface of the incident ambient and the first layer as it is plotted in Fig. 1

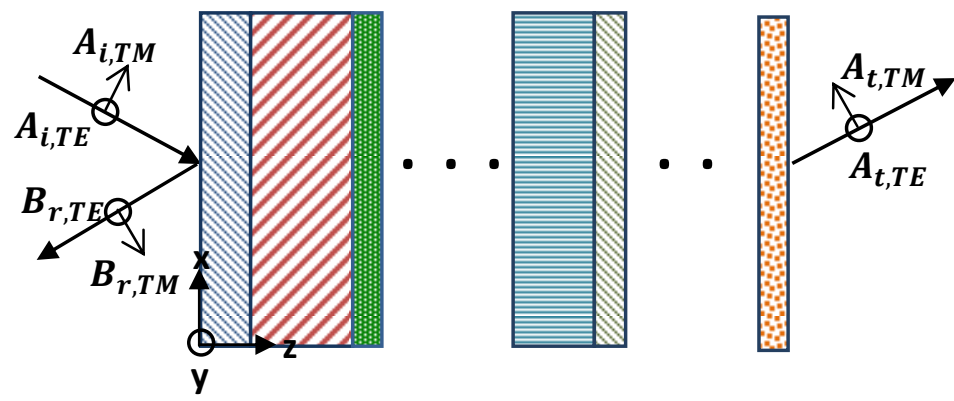

In this paper, the sign convention $e^{-i x k_{z i}}$ is chosen for propagation in $+z$ direction. The wave vector of the incident field is chosen, without loss of generality, to have $\mathrm{x}$ and $\mathrm{z}$ components. The incident wave, transmitted wave, and reflected wave, are related to each other by the transfer matrix of the layered system as

$$
\left[\begin{array}{c}
A_{i . T M} \\
B_{r, T M} \\
A_{i, T E} \\
B_{r, T E}
\end{array}\right]=T F\left[\begin{array}{c}
A_{t, T M} \\
0 \\
A_{t, T E} \\
0
\end{array}\right]
$$

TF is,

$$
T F=B C_{0}^{-1}\left(\prod_{i=1}^{N} T_{l}\left(d_{l}, \overline{\bar{\varepsilon}}_{l}\right) B C_{N}\right.
$$

Where $B C_{0}$ relates the incident and the reflected amplitudes to the tangential components of the E-field and $\mathrm{H}$-field in the incident medium and $B C_{N}$ connects the transmitted amplitude to the tangential components of the E-field and $\mathrm{H}$-field in the exit medium, then $T_{l}$ represents the transfer function of each layer, as follows to the tangential components of E-field and $\mathrm{H}$-field in the exit medium and $T_{l}$ represents the transfer function of each layer.

The flowchart of the algorithm showing the development of the transfer matrix explicitly for the $l_{t h}$ layer, is presented in figure 2. The ordered product of the layers' transfer functions from the first interface, at $z=0$, to the last interface at, $\mathrm{z}=z_{n}$, in figure 1 , results in the transfer function of the layered structure. In the absence of the surface current density and charge density at the interface regions, the tangential components of electric- and magnetic-field are continuous across the interfaces. $\mathrm{BC}_{\mathrm{l}-1}$, $\mathrm{BCl}$, and $\mathrm{BCl}_{1+1}$ in figure 2 represent the boundary condition matrixes which realize the continuity of the tangential components of the E-field and $\mathrm{H}$-field in the $\mathrm{l}-1, \mathrm{l}$, and $\mathrm{l}+1$ layers respectively. To form the $\mathrm{BC}$ matrix, four tangential components of the E-field and $\mathrm{H}$-field in each medium are required. However, since the polarization vectors of the electric field and magnetic field were found analytically the number of variables can be minimized to one. Here, the y component of the $\mathrm{H}$-field, $H_{y}$, is chosen, but others may be selected as well. As there are four different wave vectors, with the same $\mathrm{k}_{\mathrm{x}}$ value but different $\mathrm{k}_{\mathrm{z}}$ values, there are four partial fields which constitute each tangential component. The BC matrix presents the association of tangential components to the amplitude of the partial fields that constitute $H_{y}$, as shown in the following:

Figure1. Incidence, reflectance and transmittance of a plane wave 


$$
\left[\begin{array}{l}
\sum H_{y l} \\
\sum E_{x l} \\
\sum E_{y l} \\
\sum H_{x l}
\end{array}\right]=B C_{l}\left[\begin{array}{l}
H_{l 1} \\
H_{l 2} \\
H_{l 3} \\
H_{l 4}
\end{array}\right]
$$

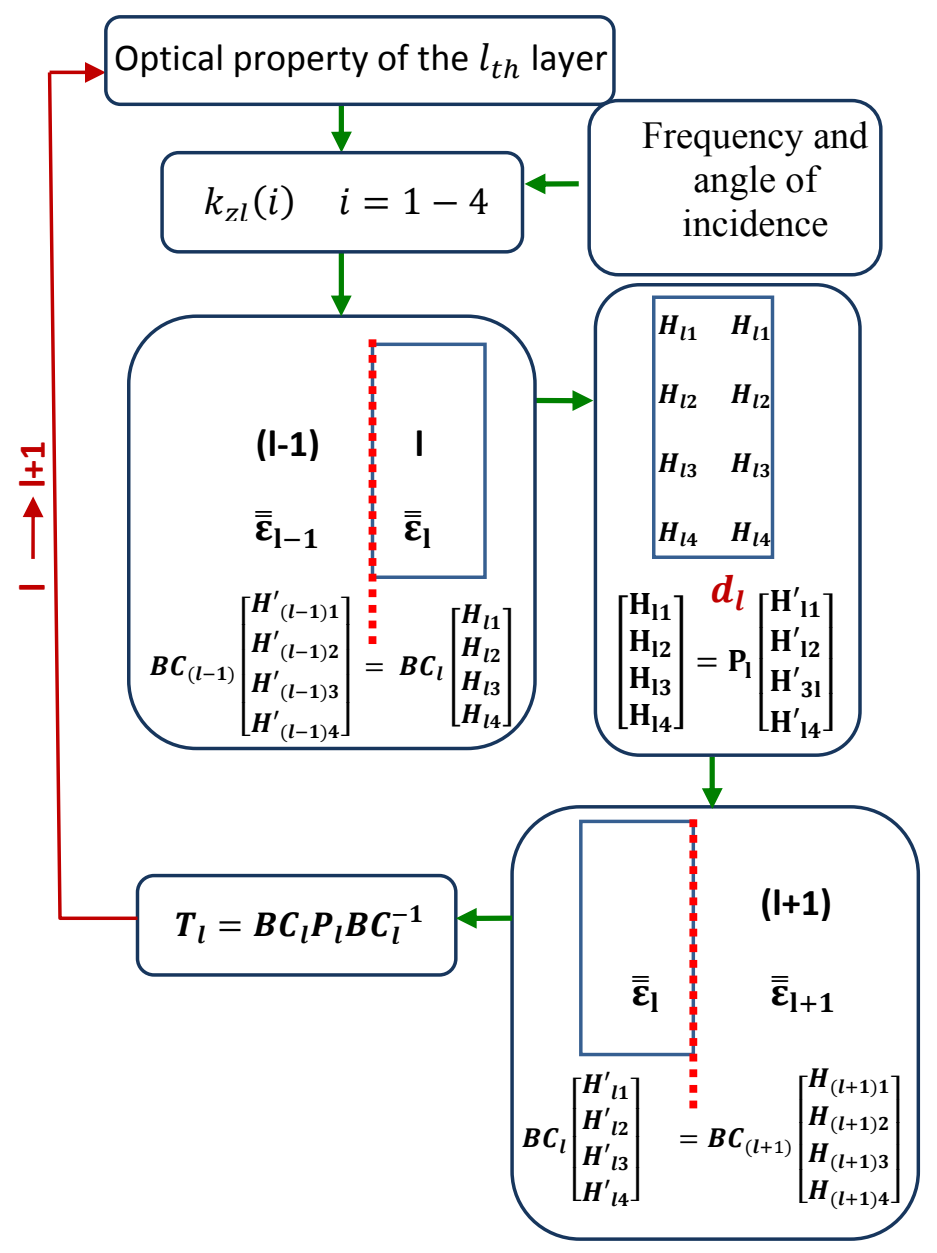

Figuer 2. The flowchart of transfer matrix development for the

$$
\text { lth layer }
$$

Where $H_{l 1}-H_{l 4}$ are the amplitudes of the four partial fields that form the $H_{y}$. The explicit expressions for the $\mathrm{BC}$ matrix are provided as follows:

$$
\begin{gathered}
B C_{l}=\left[\begin{array}{cccc}
A_{1, l} & A_{2, l} & A_{3, l} & A_{4, l} \\
1 & 1 & 1 & 1 \\
B_{1, l} & B_{2, l} & B_{3, l} & B_{4, l} \\
C_{1, l} & C_{2, l} & C_{3, l} & C_{4, l}
\end{array}\right] \\
A_{i, l}=\frac{k_{z}(i)}{\omega\left[\varepsilon_{x x}+\varepsilon_{x y}\left[\frac{\beta_{l} \lambda_{l}}{\gamma_{l} \xi_{l}}-\frac{\alpha_{l}}{\gamma_{l}}\right]-\frac{\lambda_{l} l_{\xi_{l}}}{\xi_{l}} \varepsilon_{x z}\right.} \\
B_{i, l}=\left(\frac{\beta_{l} \lambda_{l}}{\gamma_{l} \xi_{l}}-\frac{\alpha_{l}}{\gamma_{l}}\right) A_{i, l}
\end{gathered}
$$

$$
C_{i, l}=\frac{-k_{Z}(i)}{\omega \mu} B_{i, l}
$$

The forward and backward propagation coefficients of the partial fields, inside the $l_{t h}$ layer, from the $l_{t h}$ interface denoted by $\left[\begin{array}{llll}H_{l 1} & H_{l 2} & H_{3 l} & H_{l 4}\end{array}\right]^{T}$ to $(l+1)_{t h}$ denoted by $\left[\begin{array}{llll}H_{l 1}^{\prime} & H_{l 2}^{\prime} & H_{3 l}^{\prime} & H_{l 4}^{\prime}\end{array}\right]^{T}$, is shown analytically in equation (11-a) and $(11-\mathrm{b}) . d_{l}$ is the thickness of the layer and $k_{z l, i}$ is the $\mathrm{z}$ component of the wave vector in section II.

$$
\begin{gathered}
{\left[\begin{array}{l}
H_{l 1} \\
H_{l 2} \\
H_{l 3} \\
H_{l 4}
\end{array}\right]=P_{l}\left[\begin{array}{c}
H_{l 1}^{\prime} \\
H_{l 2}^{\prime} \\
H_{3 l}^{\prime} \\
H_{l 4}^{\prime}
\end{array}\right]} \\
P_{l}=\left[\begin{array}{cccc}
e^{i k_{z l, 1} d_{l}} & 0 & 0 & 0 \\
0 & e^{i k_{z l, 2} d_{l}} & 0 & 0 \\
0 & 0 & e^{i k_{z l, 3} d_{l}} & 0 \\
0 & 0 & 0 & e^{i k_{z l, 4} d_{l}}
\end{array}\right]
\end{gathered}
$$

According to figure 2, the transfer function $T_{l}$ connects the tangential components of E-field and $\mathrm{H}$-field of $(l-1)_{t h}$ layer to $(l+1)_{t h}$ layer.

$$
\left[\begin{array}{l}
\sum H_{y l-1} \\
\sum E_{x l-1} \\
\sum E_{y l-1} \\
\sum H_{x l-1}
\end{array}\right]=T_{l}\left[\begin{array}{l}
\sum H_{y l+1} \\
\sum E_{x l+1} \\
\sum E_{y l+1} \\
\sum H_{x l+1}
\end{array}\right]
$$

According to the flowchart of figure 2 and equations 10, 11, and 12 the transfer function of the $l_{t h}$ layer is

$$
T_{l}=B C_{l} P_{l} B C_{l}^{-1}
$$

The ordered product of the layers' transfer function from the first interface at $\mathrm{z}=0$ to the last interface at $\mathrm{z}=z_{n}$ connects the tangential field components at $\mathrm{z}=0$ and $\mathrm{z}=z_{n}$.

$$
T=\prod_{l=1}^{N} T_{l}
$$

The TF in equation (7) is the relation between the amplitudes of incident, reflected, and transmitted waves. In most of the cases the incident and exit medium are isotropic and $B C_{l}$ in equation (8) is simplified to,

$$
B C_{0}=\left[\begin{array}{cccc}
1 & 1 & 0 & 0 \\
\frac{k_{z 0}}{\omega \varepsilon_{0}} & -\frac{k_{z 0}}{\omega \varepsilon_{0}} & 0 & 1 \\
0 & 0 & \frac{k_{z 0}}{\omega \mu_{0}} & -\frac{k_{z 0}}{\omega \mu_{0}}
\end{array}\right]
$$




$$
B C_{N}=\left[\begin{array}{cccc}
1 & 1 & 0 & 0 \\
\frac{k_{z N}}{\omega \varepsilon_{N}} & -\frac{k_{z N}}{\omega \varepsilon_{N}} & 0 & 0 \\
0 & 0 & \frac{k_{Z N}}{\omega \mu_{N}} & -\frac{k_{z N}}{\omega \mu_{N}}
\end{array}\right]
$$

Here $k_{z 0}$ and $k_{z N}$ are the $\mathrm{z}$ components of the $\mathrm{k}$ vector in isotropic incident and exit medium respectively which become imaginary for the incident evanescent waves. In lossless medium, $k_{z 0}$ and $k_{z N}$ are real for propagating waves and imaginary for evanescent waves.

\section{Transmission of Evanescent Wave with Layered Isotropic-Anisotropic for Sub-wavelength Imaging Application}

\section{i - Transmission through a single layer of anisotropic material}

It is well-known that the resolution of the conventional optical imaging systems is restricted by the Abbe diffraction limit. The observation of the sub-wavelength details of an object is difficult due to the considerable attenuation of the scattered waves with high spatial frequency, in the nanometric vicinity of the object. An anisotropic slab with specific permittivity tensor is able to transmit the high spatial frequencies without major loss due to its hyperbolic dispersion curve [22]. Based on the transfer matrix calculation of section IV, here we show the transmission of evanescent waves from the source to image plane with an anisotropic slab. The parameters of the permittivity tensor of the slab are from [23]. As it is shown in Fig. 3, for the calculated transfer function is in excellent agreement with the data given in [23]. The anisotropic slab is nonmagnetic and described with a diagonal permittivity tensor,

$$
\overline{\bar{\varepsilon}}=\left[\begin{array}{ccc}
\varepsilon_{x x} & 0 & 0 \\
0 & \varepsilon_{y y} & 0 \\
0 & 0 & \varepsilon_{z z}
\end{array}\right]
$$

The incident wave is the TM polarized wave with magnetic field in $y$ direction. In [23] the sign of the real part of $\varepsilon_{x x}$ and $\varepsilon_{y y}$ is chosen to be positive and the real part of $\varepsilon_{z z}$ is negative, which leads to hyperbolic dispersion relation for the anisotropic slab. The $\mathrm{z}$ components of $\mathrm{k}$ vectors are,

$$
\begin{aligned}
& k_{z, 1}=-k_{z, 2}=\sqrt{k_{0}^{2} \varepsilon_{x x}-\frac{\varepsilon_{x x}}{\varepsilon_{z z}} k_{x}^{2}} \\
& k_{z, 3}=-k_{z, 4}=\sqrt{\varepsilon_{y y} k_{0}^{2}-k_{x}^{2}}
\end{aligned}
$$

The dispersion relation (22-a) shows that for the opposite signs of $\varepsilon_{x x}$ and $\varepsilon_{z z}$, the waves with large spatial frequencies, $k_{x}$, will not decay in the anisotropic medium $\left(\mathrm{k}_{\mathrm{zi}}\right.$ remains real). According to the expressions in section IV the transfer function for the TM incident wave is

$$
T_{T M}=\frac{C_{t, T M}}{A_{i, T M}}=\frac{\mathrm{T}_{33}}{\mathrm{~T}_{11} \mathrm{~T}_{33}-\mathrm{T}_{13} \mathrm{~T}_{31}}
$$

Figure 3 demonstrates the transfer function of an anisotropic slab in interaction with propagating $\left(k_{x}=0-\right.$ $\left.k_{0}\right)$ incident waves and evanescent $\left(k_{x}=k_{0}-10 k_{0}\right)$ incident, the thickness of the slab in $400 \mathrm{~nm}$ and the wavelength of incident wave $700 \mathrm{~nm}$.According to the figure 3 , the anisotropic slab with specific permittivity tensor is capable to preserve the evanescent waves, which can be a considerable achievement in sub-wavelength imaging techniques. Having the explicit expressions for the transfer function facilitates one to study the destructive and constructive effects i.e. loss, thickness, and operating frequency, precisely and design an optimum imaging device in this case. As an example in Fig. 4, sub-wavelength imaging with an anisotropic slab for two Gaussian pulses separated $23 \mathrm{~nm}$ is realized. The monochromatic wave is illuminated with the wavelength of $700 \mathrm{~nm}$ which result in $\sim \frac{\lambda}{35}$ sub-wavelength resolution.

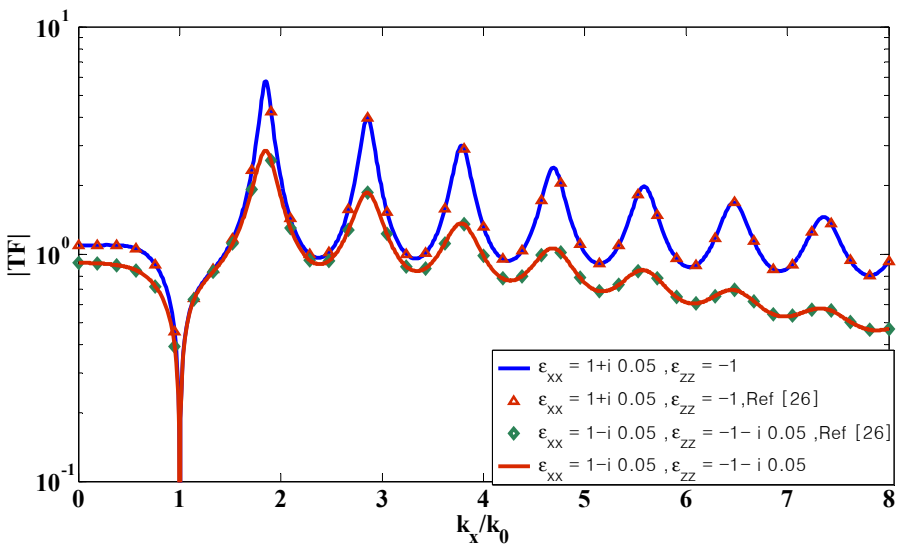

Figure 3 .Transfer functions for lossy and optically active anisotropic slab

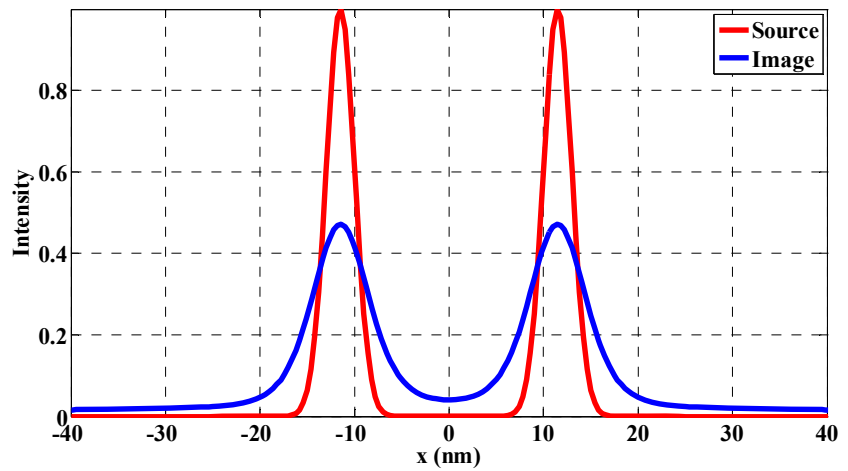

Figure 4. Sub-wavelength imaging with an anisotropic slab $\varepsilon_{x x}=\varepsilon_{y y}=1-j .02 \varepsilon_{z z}=-1, \mathrm{~d}=400 \mathrm{~nm}, \lambda=700 \mathrm{~nm}$ 
In sub-wavelength imaging systems with isotropic metal layer based superlenses, the evanescent waves grow exponentially until a particular film thickness thereafter; the material loss becomes more prominent [24]. Hence, the multilayer lens is greatly advantageous to establish a feasible spacing between the sample and its image. Based on the analytical algorithm derived in section III, here the sub-wavelength imaging with the layered isotropicanisotropic structure is shown. The layered structure is made of successive arrangement of anisotropic layers and isotropic slabs. In conventional layered superlens with metallic and dielectric slabs, the optimal imaging occurs when the overall thickness of metal slabs and dielectric slabs are equal. However for the layered isotropicanisotropic structure, this ratio is not required due to the different imaging mechanism. In metal-dielectric lenses the sub-wavelength imaging is possible due to the amplification of evanescent waves while the sub-wavelength imaging in anisotropic-isotropic layers is due to the preservation of evanescent waves.
Figure 6, shows the realization of sub-wavelength imaging for two Gaussian pulses which are $40 \mathrm{~nm}$ away at $\lambda=$ $500 \mathrm{~nm}$ and $\lambda=700 \mathrm{~nm}$ which result in the sub-wavelength resolution of $\frac{\lambda}{12.5}$ and $\frac{\lambda}{17.5}$ respectively, far beyond the diffraction limit.

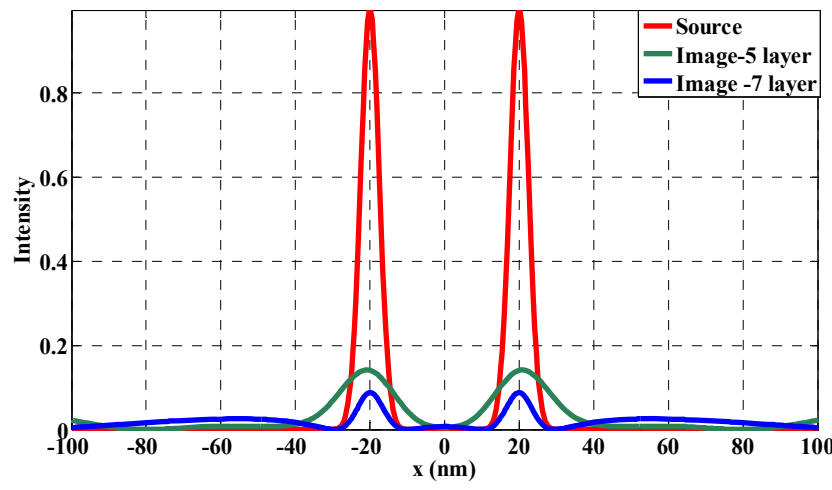

Figure 6. Sub-wavelength imaging with layered anisotropicisotropic lens

Table 1. Specifications of 5 layer and 7 layer superlens

\begin{tabular}{cccc}
\hline Number of layers & Wavelength(nm) & Thickness $(\mathrm{nm})$ & Optical Property \\
\hline 5 & 500 & 40044004400 & $\varepsilon_{x x}=2-i .05, \varepsilon_{y y}=2-i .05, \varepsilon_{z z}=-2$ \\
7 & 700 & 100210021002100 & $\varepsilon_{x x}=1-i .02, \varepsilon_{y y}=1-i .02, \varepsilon_{z z}=-1$ \\
\hline
\end{tabular}

Consequently the isotropic layers can be chosen as thin as possible to minimize the attenuation of the evanescent waves which result in higher resolution compared to only isotropic multilayer designs. Figure 5 demonstrates the transfer function of two different arrangements for layered structures, the specifications of each structure is provided in table 1.

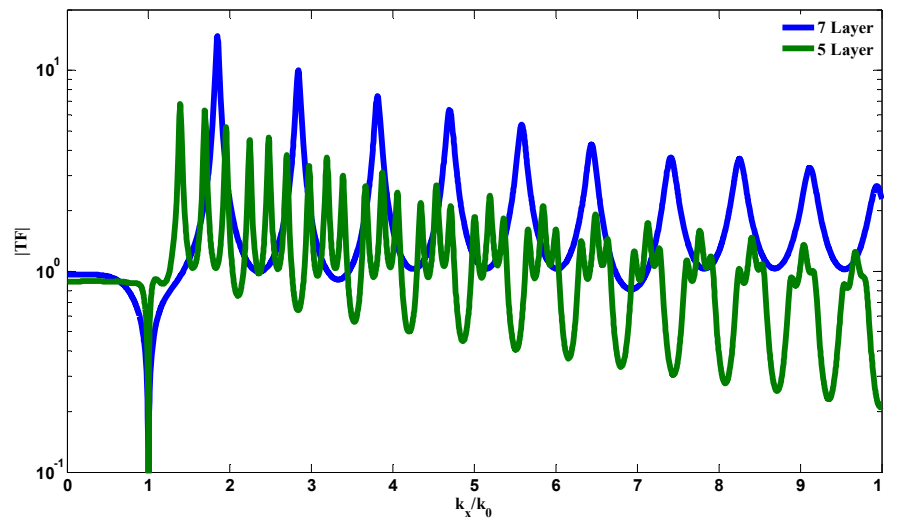

Figure 5. Transfer function for layered anisotropic-isotropic lens, the specifications of the layered strictures are provided in table 1

\section{The effect of gyrotropy on Sub-wavelength imaging}

Recently, it is shown that magnetized plasma with appropriately designed parameters supports subdiffraction propagation of electromagnetic waves along the direction of the applied magnetic field [25]. The electromagnetic properties of magnetized plasma can be tuned by varying the external magnetic field or the density of the plasma. Under strong external DC magnetic field, the plasma exhibits the following dielectric function,

$$
\varepsilon=\left[\begin{array}{ccc}
\varepsilon_{x x} & -i \varepsilon_{g} & 0 \\
i \varepsilon_{g} & \varepsilon_{x x} & 0 \\
0 & 0 & \varepsilon_{z z}
\end{array}\right]
$$

where $\varepsilon_{x x}=1+\frac{\omega_{p}^{2}}{\omega_{c}^{2}-\omega^{2}}, \varepsilon_{g}=1+\frac{\omega_{p}^{2} \omega_{c}}{\omega\left(\omega_{c}^{2}-\omega^{2}\right)}$ and $\varepsilon_{z z}=1-\frac{\omega_{p}^{2}}{\omega^{2}}$ $[25], \omega_{p}$ is the plasma frequency and $\omega_{c}$ is the cyclotron frequency. In this section the effect of off-diagonal element, $\varepsilon_{g}$, is investigated on the quality of subwavelength imaging in layered structure. The four explicit expressions for the normal component of propagation vector are as follows, 
$k_{z, 1}=-k_{z, 2}$

$\sqrt{\frac{\left(\varepsilon_{x x}+\varepsilon_{z z}\right) k_{x}^{2}-2 \varepsilon_{x x} \varepsilon_{z z} k_{0}^{2}+\sqrt{\left(\varepsilon_{x x} k_{x}^{2}-\varepsilon_{z z} k_{x x}^{2}\right)^{2}+4 \varepsilon_{z z} \varepsilon_{g}^{2}\left(\varepsilon_{z z} k_{0}^{2}-k_{x}^{2}\right)}}{-2 \varepsilon_{z z}}}$

$k_{z, 3}=-k_{z, 4}$

$$
\sqrt{\frac{2 \varepsilon_{x x} \varepsilon_{z z} k_{0}^{2}-\left(\varepsilon_{x x}+\varepsilon_{z z}\right) k_{x}^{2}+\sqrt{\left(\varepsilon_{x x} k_{x}^{2}-\varepsilon_{z z} k_{x}^{2}\right)^{2}+4 \varepsilon_{z z} \varepsilon_{g}^{2}\left(\varepsilon_{z z} k_{0}^{2}-k_{x}^{2}\right)}}{2 \varepsilon_{z z}}}
$$

Note that the dispersion relations (24-a) and (24-b) are simplified to equation $(22-\mathrm{a})$ and $(22-\mathrm{b})$, when $\varepsilon_{g}$ is set to zero. The overall quality of imaging depends on how well the large wave vectors are transferred through the layered structure. Figure 7(a) shows the normal components of the wave vector $k_{z, 1}, k_{z, 3}$ in function of $k_{x}$ and $\varepsilon_{g}$, when the diagonal elements of the tensor are $\varepsilon_{x}=1-i 0.02$ and $\varepsilon_{z}=$ $-1, k_{z, 2}, k_{z, 4}$ are different from $k_{z, 1}, k_{z, 3}$ only in a negative sign, equation (24). For reference, figure $7(\mathrm{~b})$ presents the normal components of the wave vector in the in the absence of $\varepsilon_{g}$ which is the material with diagonal permittivity tensor.
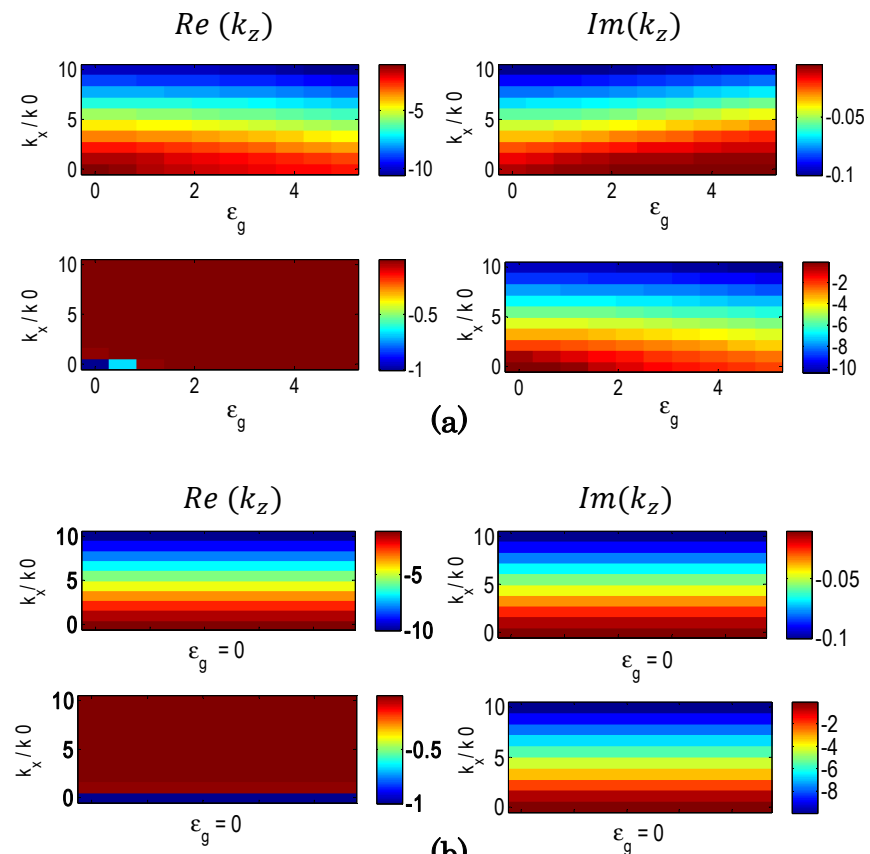

(b)

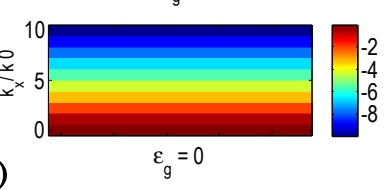

Figure 7 Normal component of $\mathrm{k}$ vector as function of $k_{x}$ and $\varepsilon_{g}$ (a) Gyrotropic dielectric function (b) Diagonally anisotropic dielectric function

A it can be observed in figure 7 , the off-diagonal elements of the electric permittivity tensor in case of gyrotropic media provides additional degree of freedom compared to the anisotropic case to engineer the normal components of the wave vector. As there are four partial waves, which contribute to the transmission and reflection of harmonics with different wave vectors, it is not obvious whether this variation is constructive or destructive. Therefore the quality of image is investigated in function of the offdiagonal elements $\varepsilon_{g}$ for the superlens made of 7 layers as specified in table 1 . Figure 8 plots the transfer function of the layered structure for several values of the off-diagonal tensor element $\varepsilon_{g}$, while figure 9 presents the corresponding intensity distributions in the image plane.

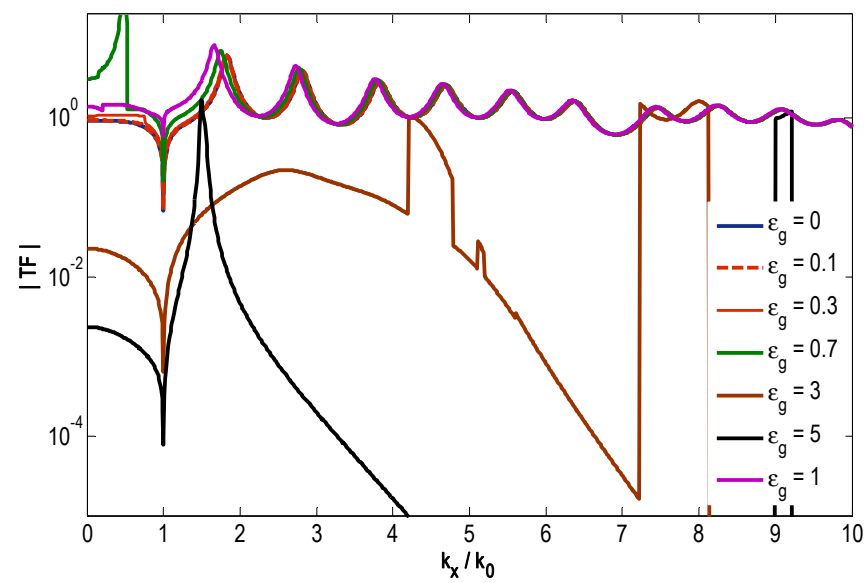

Figure 8 Transfer function of the 7 layer thick superlens for different values of the off-diagonal tensor element . $\varepsilon_{g}$

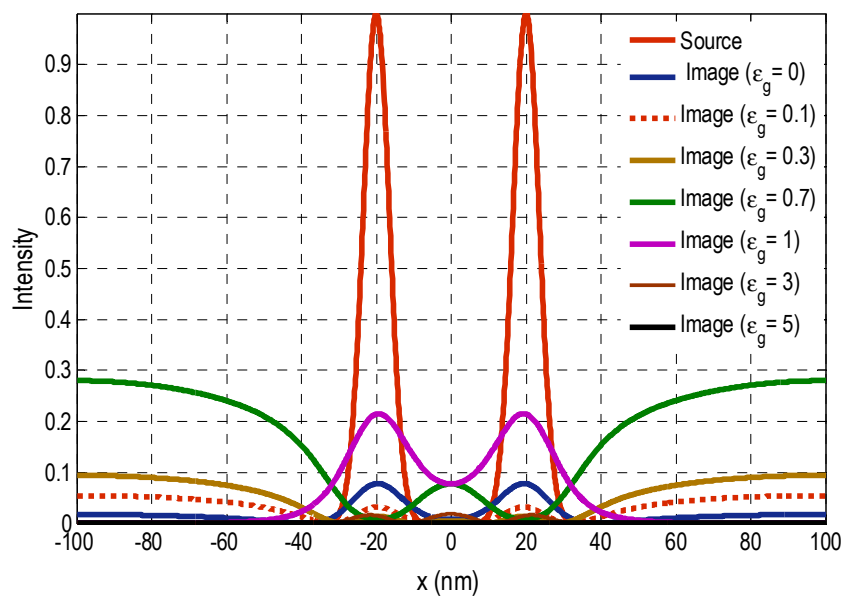

Figure 9 Sub-wavelength imaging with layered gyrotropicisotropic lens

As it is shown in figure 9 , the effect of gyrotropy can be constructive $\left(\varepsilon_{g}=1\right)$ or destructive $\left(\varepsilon_{g}=0.1,0.3,0.7,3,5\right)$. It is also shown in [25] that for external DC magnetic field of 1 and $2 \mathrm{~T}$, the imaging process is not satisfactorily, while applying DC magnetic field of 3 and $4 \mathrm{~T}$ results in the formation of image with sub-wavelength features. Consequently carefully designed gyrotropic permittivity tensor results in dispersion curves required for improved subwavelength imaging. It should be noted that the optimum value of $\varepsilon_{g}$ depends on the other elements of the permittivity tensor as well. For instance, figure 10 shows 
the imaging features for two layered superlenes with identical geometry and the same value of the off-diagonal tensor element $\varepsilon_{g}$, while the diagonal elements of the permittivity tensor differ (see table 2). Consequently, in case of gyrotropic materials, the sub-wavelength imaging can be improved by relative adjustment of the diagonal elements of the permittivity tensor.

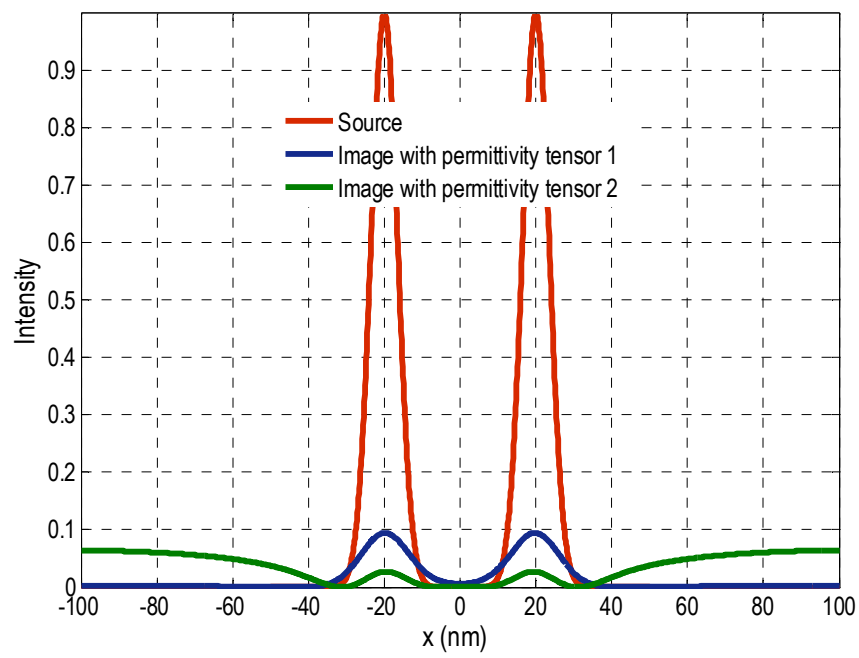

Figure 10 Imaging with gyrotropic layered superlens varying the diagonal elements of the dielectric tensor

\section{Acknowledgement}

This research is supported by the Agency for Science and Technology Research (A*STAR), Singapore.

\section{Conclusion}

The explicit expressions are derived for the polarizations of the electric and magnetic field in general anisotropic medium. Based on the vector fields' polarizations, a procedure is introduced to calculate the transmission matrix for the structure with arbirary numbers of anisotropic layers .In addition, it is shown that the provided solution is also suitable for propagation of monochromatic wave in isotropic medium and an explicit exprassion for transfer function of isotropic medium which is compatible with $4 \times 4$ matrix algebra is presented. The $4 \times 4$ matrixes which connect the amplitudes of the waves in incident and exit medium, to the tangential fields' components are provided for both propagating and evanescent waves. Based on the presented explicit solution, it is shown that the single layer and multilayer anisotropic structure is capable of deep sub-wavelength imaging. For the anisotropic layer with the specified optical properties, the provided analytical solutions for $k_{z}$ prove the transmission of the evanescent waves through the layered structure, which results in increase of the resolution much beyond the diffraction limit. The effect of gyrotropy on sub-wavelength imaging is investigated and it is shown that based on the value of offdiagonal parameter of the permittivity tensor, gyrotropy might has constructive or destructive effect on imaging. Hence, the carefully designed permittivity tensors with gyrotropy are promising for advancement in subwavelength imaging.

Table 2. Specifications of layered superlens

\begin{tabular}{|c|c|c|c|c|}
\hline & Number of layers & Wavelength(nm & Thickness(nm) & Optical Property \\
\hline (1) & 7 & 700 & 00210021002100 & $\varepsilon_{x x}=2-i .05, \varepsilon_{y y}=2-i .05, \varepsilon_{z z}=-2 \varepsilon_{g}=0.3$ \\
\hline (2) & 7 & 700 & 100210021002100 & $\varepsilon_{x x}=1-i .05, \varepsilon_{y y}=1-i .05, \varepsilon_{z z}=-1 \varepsilon_{g}=0.3$ \\
\hline
\end{tabular}


The general solution for the wave propagation in anisotropic media presented in [12,14], fails for the isotropic case. Here we use the expressions provided for the polarizations of E-field and $\mathrm{H}$-field in equations (4-6), and propagation matrix given in equation (11) to derive the expression of the transfer function for isotropic layer. As the transfer function of each layer connects the tangential components of $\mathrm{E}$-fields and $\mathrm{H}$-fields across the two interfaces of the layer and, equations (6-8) provides the relations between the tangential components,

$$
\left[\begin{array}{c}
H_{y} \\
E_{x} \\
E_{y} \\
H_{x}
\end{array}\right]=\left[\begin{array}{cccc}
1 & \frac{k_{z l}}{\omega \varepsilon_{l}} & 0 & 0 \\
\frac{k_{z l}}{\omega \varepsilon_{l}} & 1 & 0 & 0 \\
0 & 0 & 1 & \frac{\omega \mu_{l}}{k_{z l}} \\
0 & 0 & \frac{k_{z l}}{\omega \mu_{l}} & 1
\end{array}\right]\left[\begin{array}{c}
H_{y} \\
E_{x} \\
E_{y} \\
H_{x}
\end{array}\right]
$$

As it is expected $H_{y}$ and $E_{x}$ are not coupled to $E_{y}$ and $H_{x}$ anymore and wave propagation in the isotropic layer can be decomposed for TE and TM waves. The $4 \times 4$ boundary condition matrix and propagation matrix is then reduced to two $2 \times 2$ matrices as

$$
\begin{aligned}
B C_{l, T M} & =\left[\begin{array}{cc}
1 & 1 \\
\frac{k_{z l}}{\omega \varepsilon_{l}} & -\frac{k_{z l}}{\omega \varepsilon_{l}}
\end{array}\right] \\
T_{l, i s o} & =\left[\begin{array}{cc}
\cos \left(k_{z l} d_{l}\right) & i \frac{\omega \varepsilon_{l}}{k_{z l}} \sin \left(k_{z l} d_{l}\right) \\
i \frac{k_{z l}}{\omega \varepsilon_{l}} \sin \left(k_{z l} d_{l}\right) & \cos \left(k_{z l} d_{l}\right) \\
0 & 0 \\
0 & 0
\end{array}\right.
\end{aligned}
$$

$$
\begin{gathered}
B C_{l, T E}=\left[\begin{array}{cc}
1 & 1 \\
\frac{k_{z l}}{\omega \mu_{l}} & -\frac{k_{z l}}{\omega \mu_{l}}
\end{array}\right] \\
P_{l, T M}=P_{l, T E}=\left[\begin{array}{cc}
e^{i k_{z l d_{l}}} & 0 \\
0 & e^{-i k_{z l d_{l}}}
\end{array}\right]
\end{gathered}
$$

The propagation matrix in equation (A4) shows that in the isotropic layer, there are two partial waves, one propagates forward and the other propagates backward, which is in agreement with the number and sign of eigenvectors of the wave equation matrix for the isotropic medium. Following the same procedure as presented in figure 2 , the $4 \times 4$ matrix for the transfer function, which is compatible with the calculations of the anisotropic system is

$$
T_{l, i s o}=B C_{l . i s o} P_{l, i s o} B C_{l, i s o}^{-1}
$$

Where, $B C_{l, \text { iso }}=\left[\begin{array}{cc}B C_{l, T M} & {[0]} \\ {[0]} & B C_{l, T E}\end{array}\right] \operatorname{and} P_{l, \text { iso }}=\left[\begin{array}{cc}P_{l, T M} & {[0]} \\ {[0]} & P_{l, T E}\end{array}\right]$. The explicit expression for the transfer matrix on the isotropic slab is ,

$$
\left.\begin{array}{cc}
0 & 0 \\
0 & 0 \\
\cos \left(k_{z l} d_{l}\right) & -i \frac{\omega \mu_{l}}{k_{z l}} \sin \left(k_{z l} d_{l}\right) \\
-i \frac{k_{z l}}{\omega \mu_{l}} \sin \left(k_{z l} d_{l}\right) & \cos \left(k_{z l} d_{l}\right)
\end{array}\right]
$$




\section{Reference}

[1] J. Diener, D. I. Kovalev, N. Kuenzner, , E. Gross, G. Polisski, F. Koch, M. Fujii, "Spatially nanostructured silicon for optical applications," In Proceeding of Sixth International Conference on Material Science and Material Properties for Infrared Optoelectronics, 12-22 ( 2003).

[2] Q. D. Nguyen, "Electrochemistry in anisotropic etching of silicon in alkaline solutions: a kinetic wave analysis," UT publication (2007).

[3] H. W. Park, T. Kim, J. Huh, M. Kang, J. E. Lee, H. Yoon, "Anisotropic Growth Control of Polyaniline Nanostructures and Their Morphology-Dependent Electrochemical Characteristics," ACS Nano,6,7624-7633 (2012)

[4] Y. Hanazaki, J. Masumoto, S. Sato, K. Furusawa, A. Fukui, ,N. Sasaki, "Multiscale analysis of changes in an anisotropic collagen gel structure by culturing osteoblasts," ACS Applied Materials \& Interfaces (2013).

[5] K. D. Costa, E. J. Lee, J. W. Holmes, "Creating alignment and anisotropy in engineered heart tissue: role of boundary conditions in a model three-dimensional culture system ," Tissue Eng. 567-77 (2003)

[6] A. N. Parikh, D. L. Allara, " Quantitative determination of molecular structure in multilayered thin films of biaxial and lower symmetry from photon spectroscopies. I. Reflection infrared vibrational spectroscopy," The Journal of chem. phys., 96 (1992)

[7] J. Schesser, G. Eichmann," Propagation of plane waves in biaxially anisotropic layered media," J.Opt.Soc.Am 62. 786-791 (1972).

[8] J. J. Stamnes , G. C. Sherman, "Reflection and refraction of an arbitrary wave at a plane interface separating two uniaxial crystals,” J. Opt. Soc. Am. 67, 683-695 (1977).

[9] J. Stamnes, G. S. Sithambaranathan," Reflection and refraction of an arbitrary electromagnetic wave at a plane interface separating anisotropic and a biaxial medium," J. Opt. Soc. Am. A 22, 3119-3129 (2001).

[10] R. A. Farrell, D. Rouseff, R. L. McCally," Propagation of polarized light through two- and three-layer anisotropic stacks," J. Opt. Soc. Am. A, 22, 1981-1992 (2005).

[11] X. Yang, Y. Jia, Y. Zhao, T. Zhang, X. Zhu," Dispersion of linearly polarized light propagating in a thin birefringent plate," J. Opt. Soc. Am. A 22,752-759 (2005).
[12] P. Yeh, "Electromagnetic propagation in birefringent layered media," J. Opt. Soc. Am. 69, 742-755 (1979).

[13]D. W. Berreman,"Optics in Stratified and Anisotropic Media: 4 × 4-Matrix Formulation,” J. Opt. Soc. Am. 62, 502-510 (1972)

[[14] M. Schubert, "Polarization-dependent optical parameters of arbitrarily anisotropic homogeneous layered systems," Phys. Rev. B 53, 4265-4274 (1996)

[15] N. Ouchani,D. Bria, B. Djafari-Rouhani,A. Nougaoui," Transverse-electric/transverse-magnetic polarization converter using $1 \mathrm{D}$ finite biaxial photonic crystal," J. Opt. Soc. Am 24, 2710-2718 (2007).

[16] M. Sluijter, D.K. de Boer, J.J. Braat," General polarized raytracing method for inhomogeneous uniaxially anisotropic media," J. Opt. Soc. Am. A 25, 1260-1273 (2008).

[17] P. Ginzburg, F. J. Fortuño, G. A. Wurtz, W. Dickson ,"Manipulating polarization of light with ultrathin epsilon-near-zero metamaterials," Optics Express, Vol. 21, Issue 12, 14907-14917 ( 2013).

[18] D. Mounier, P. Picart, P. Babilotte, P. Ruello, ”Jones matrix formalism for the theory of picosecond shear acoustic pulse detection, Optics Express, Vol. 18, Issue 7, 6767-6778 (2010)

[19] D. Sun, J. Rioux, J. E. Sipe, Y. Zou, M. T. Mihnev, C. Berger, T. B. Noris, "Evidence for interlayer electronic coupling in multilayer epitaxial graphene from polarization-dependent coherently controlled photocurrent generation," Physical Review 16,Vol. B85 (2012)

[20] I. V. Lindell, F. Olyslager, "Generalized decomposition of electromagnetic media," Antenna and propagation IEE ,46 (1998).

[21] E. Langereis, S. B. S. Heil, H. C. M. Knoops, W. Keuning, M. C. M. Van de Sanden, W. M. M. Kessels, "In situ spectroscopic ellipsometry as a versatile tool for studying atomic layer deposition," Journal of Physics D: Applied Physics, Vol 42, 073001 (2009).

[22] A. Salandrino, N. Engheta, "Far-field subdiffraction optical microscopy using metamaterial crystals: Theory and simulations," Phys.Rev. B 7, Vol.74, 075103 (2006)

[23] H. Liu, K. J. Webb," Sub-wavelength imaging opportunities with planar uniaxial anisotropic lenses", Optics Letters, Vol. 33, Issue 21, 2568-2570 (2008).

[24] N. Fang, H. Lee, C. Sun, and X. Zhang, "Sub-diffraction-limited optical imaging with a silver superlens," Science 5721, 308, ,534-537 (2005).

[25] S. Zhang, Y. Xiong, G. Bartal, X. Yin, X. Zhang, "Magnetized plasma for reconfigurable subdiffraction imaging," Phys. Rev. Lett. 24, Vol. 106, 243901 (2011) 